\title{
BMJ Open Comparison of general anaesthesia and regional anaesthesia in terms of mortality and complications in elderly patients with hip fracture: a nationwide population-based study
}

\author{
Eun Jin Ahn, ${ }^{1}$ Hyo Jin Kim, ${ }^{1}$ Kyung Woo Kim, ${ }^{1}$ Hey Ran Choi, ${ }^{1}$ Hyun Kang, ${ }^{2}$ \\ Si Ra Bang ${ }^{1}$
}

To cite: Ahn EJ, Kim HJ, Kim KW, et al. Comparison of general anaesthesia and regional anaesthesia in terms of mortality and complications in elderly patients with hip fracture: a nationwide populationbased study. BMJ Open 2019;9:e029245. doi:10.1136/ bmjopen-2019-029245

- Prepublication history for this paper is available online. To view these files, please visit the journal online (http://dx.doi. org/10.1136/bmjopen-2019029245).

This work was presented at the 2018 World Congress on Regional Anesthesia \& Pain Medicine in New York, 19-21 April 2018, as a Best of a Meeting Award.

Received 21 January 2019 Revised 07 August 2019 Accepted 09 August 2019

Check for updates

(c) Author(s) (or their employer(s)) 2019. Re-use permitted under CC BY-NC. No commercial re-use. See rights and permissions. Published by BMJ.

For numbered affiliations see end of article.

Correspondence to

Dr Si Ra Bang;

sira1045@naver.com

\section{ABSTRACT}

Objective To evaluate the effects of anaesthesia on postoperative outcome in elderly patients who underwent hip fracture surgery.

Setting Nationwide National Health Insurance Sharing Service database of Korea.

Participants All patients aged $\geq 65$ years old who underwent hip fracture surgery, covered by the Korean National Health Insurance, between 1 January 2009 and 31 December 2015.

Interventions Hip fracture surgery under general anaesthesia (group GA) or regional anaesthesia (group RA), with a principal diagnosis of femoral fracture.

Primary and secondary outcome measures The primary outcome was the anaesthetic-type effect on 30-day mortality and the secondary outcome was postoperative delirium requiring pharmacological intervention.

Results Among the 96289 patients who underwent hip fracture surgery, 25593 and 70696 patients received GA and RA, respectively. After propensity score matching, 25593 remained in each group. Postmatching mortality was lower in the RA than in the GA group (574 (2.24\%) vs $654(2.55 \%), \mathrm{p}=0.0047,95 \% \mathrm{Cl}-0.0099$ to 0.0159$)$. Delirium incidence was lower in the RA than in the GA group (5187 (20.27\%) vs 5828 (22.77\%), p<0.0001, $95 \% \mathrm{Cl} 0.019$ to 0.045$)$. The incidence of intensive care unit stay and ventilator care was lower in the RA than in the GA group (5838 (22.1\%) vs 8055 (31.47\%), $\mathrm{p}<0.0001$, $95 \% \mathrm{Cl} 0.046$ to 0.070 and $459(1.73 \%)$ vs 1207 (4.72\%), $\mathrm{p}<0.0001,95 \% \mathrm{Cl}-0.0024$ to 0.023 , respectively). Conclusion RA was associated with better outcomes than GA, in terms of mortality, delirium, intensive care unit admission and ventilator care, in elderly patients who underwent hip fracture surgery.

\section{INTRODUCTION}

The ageing population is rapidly growing, particularly in developed countries. South Korea is rapidly turning into an aged society, defined as a society with the elderly population exceeding $14 \%$ of the total population. ${ }^{1}$
Strengths and limitations of this study

- It was based on a large and representative national database that is widely used as a reliable research tool for identifying trends and demographics.

- We used more recent data (2009-2015) to reflect improvements in anaesthetic drugs, perioperative haemodynamic monitoring and perioperative pain management.

- The database did not include clinical data, and it may have contained coding errors.

Ha $e t a l^{2}$ reported that the incidence of hip fracture is continually increasing and that the predicted increases will cause a serious socioeconomic burden and a challenge for public healthcare management in Korea. Hip fractures are usually associated with adverse complications, including mortality, which lead to a medical and financial burden on society. ${ }^{3}$

One of the most common adverse complications of hip fracture in elderly patients is delirium. It is associated with an increased rate of cognitive decline, institutionalisation and mortality. ${ }^{4}$ To decrease the mortality rate and postoperative delirium, a careful consideration of the type of anaesthesia used in hip fracture surgery is recommended, although the effects of anaesthesia type on mortality and outcome in geriatric patients are debated. Some reports have stated that most anaesthesia-related complications are multifactorial. However, in hip fracture surgery, it has been reported that regional anaesthesia (RA) yields a more favourable outcome.

In this study, we used a nationwide database to evaluate the effects of anaesthesia on postoperative outcome in elderly patients who underwent hip fracture surgery. The primary 
outcome of this study was the effect of the anaesthetic type on the 30-day mortality, and the secondary outcome was postoperative delirium requiring pharmacological intervention.

\section{METHODS}

The need to obtain informed consent was waived because we used deidentified administrative data. The study population consisted of all patients 65 years old or older, who underwent hip fracture surgery, covered by the Korean National Health Insurance Service (NHIS), between 1 January 2009 and 31 December 2015.

The NHIS is a single health insurer, managed by the Korean government, which covers approximately $97 \%$ of Koreans, while the remaining $3 \%$ of Koreans who cannot afford national insurance are covered by the Medical Aid Program (MAP). ${ }^{5}$ Claims submitted for reimbursement to NHIS and MAP are reviewed by the Health Insurance Review and Assessment Service, a central office in the Korean Ministry of Health. Our data were obtained from the National Health Information Database (NHID), created by the NHIS. The NHID is a public database of healthcare utilisation, health screening, sociodemographic variables and mortality for the entire population of South Korea and comprises data obtained between 2002 and 2015. The NHID can be accessed by the researchers whose study protocols are approved by the official review committee. Moreover, this study conformed to the Strengthening the Reporting of Observational Studies in Epidemiology guidelines for reporting observational cohort studies (www.strobe-statement.org).

\section{Patient and public involvement}

No patients or members of the public were involved in the design of this study.

\section{Participants}

We included all patients 65 years old or older, who underwent hip surgery in hospitals in Korea between 1 January 2009 and 31 December 2015. The study period was based on admission date. The major inclusion criterion was admission with a principal diagnosis of femoral neck fracture (S720) and trochanteric fracture (S721), as identified from the patient's overall diagnosis based on the

code. We included the patients who were admitted with at least one of the following surgical operations based on procedure codes: open reduction of fractured extremity (femur), total arthroplasty (hip), hemiarthroplasty (hip) and revision of hemiarthroplasty (hip). We included patients who received general, spinal and epidural anaesthesia. We excluded patients who received both GA and RA. Patients with a diagnosis of multiple trauma or fracture ('S00-S70', 'S73-99', 'T07', 'T14') were excluded. Additionally, we excluded patients who underwent more than two such operations during the same period of admission. Thirty-day mortality was defined as death either inside or outside the hospital due to any cause within 30 days of admission (in the hospital or after discharge).

\section{Independent variables}

Patients were classified into two groups according to the specific anaesthesia type (general anaesthesia (GA) or RA). The RA group was administered epidural anaesthesia, spinal anaesthesia or both. The patient characteristics that were recorded were sex, age, comorbid conditions and hospital type (medical centre, general hospital or clinic). The Elixhauser comorbidity method, which outperforms the Charlson Index in predicting hospital inpatient death after orthopaedic surgery, was used to identify the patients' comorbidities. ${ }^{6}$ The Elixhauser comorbidity method uses the sum of weighted points based on the presence or absence of 31 different medical conditions (including congestive heart failure, cardiac arrhythmias, valvular disease, pulmonary circulation disorders, peripheral vascular disorders, uncomplicated hypertension, complicated hypertension, paralysis, other neurological disorders, chronic pulmonary disease, uncomplicated diabetes mellitus, complicated diabetes mellitus, hypothyroidism, renal failure, liver disease, peptic ulcer disease, AIDS/HIV infection, lymphoma, metastatic cancer, solid tumour without metastasis, rheumatoid arthritis, coagulopathy, obesity, weight loss, fluid and electrolyte disorders, blood loss anaemia, deficiency anaemia, alcohol abuse, drug abuse, psychoses and depression). The hospital type was classified, according to the number of beds, into a medical centre, general hospital or clinic. Stay in the intensive care unit (ICU), ventilator care and cost during the hospital stay were also recorded.

The primary outcome was the 30-day mortality (defined as death due to any cause within 30 days of the indexed date, inside or outside the hospital). As a secondary outcome, we used the occurrence of delirium, defined by a record of intravenous administration of haloperidol, risperidone and quetiapine at least once during the hospital stay period.

Other complications, such as myocardial infarction, pulmonary embolism, cerebral haemorrhage, acute respiratory distress syndrome, pulmonary oedema, acute kidney injury, hepatic failure and sepsis, were also recorded.

\section{Statistical analysis}

To reduce selection bias and the potential baseline differences between the RA and GA groups, prior propensity score matching (PSM) was performed via the calliper matching method to match patients from the two groups in a 1:1 ratio. The propensity score was calculated by logistic regression analysis using the following covariates: age, sex, hospital type and comorbid conditions using the Elixhauser method to examine the association of anaesthesia type with 30-day mortality, other complications and delirium after hip fracture surgery. 


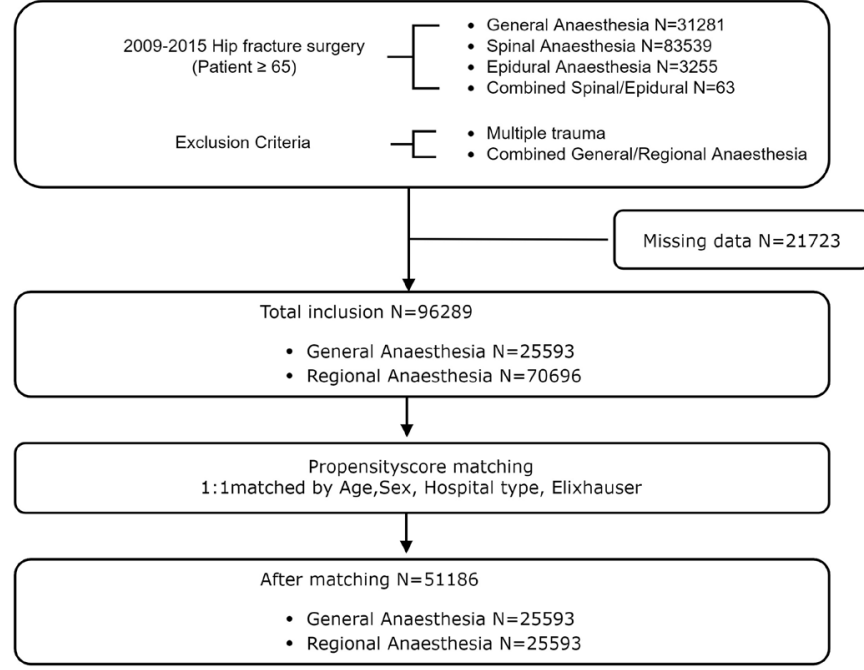

Figure 1 Flow diagram of included patient numbers.

Prior to PSM, patient characteristics were summarised using descriptive statistics. For continuous variables, data are presented as the median (range), and groups were compared using the Mann-Whitney U test. Descriptive variables were analysed using $\chi^{2}$ analysis. Postmatching continuous variables are presented as the median (range); these were compared using Wilcoxon's signed rank-sum test. Categorical variables are presented as absolute numbers (percentages). Statistical differences between groups were tested with McNemar's tests. A p value $<0.05$ was considered statistically significant. SAS V.9.3 was used for the analysis. All statistical testing was two sided at a significance level of 0.05 .

\section{RESULTS}

We identified 118012 patients undergoing hip fracture surgery, who were 65 years old or older and who were admitted to the hospital during 2009-2015; of these, 63 patients were excluded due to duplication of patient data, as they underwent combined spinal and epidural anaesthesia, and 21723 patients were excluded due to missing data. Finally, 96289 patients were included in our study. Among these, 25593 patients were included in the GA group and 70696 patients were included in the RA group (figure 1). Before PSM, baseline characteristics, including age, sex, comorbid conditions and hospital type, differed between the RA and GA groups (table 1). After performing 1:1 PSM, 25593 patients were included in each group, with no significant difference between the two groups in terms of covariates, including age, sex, hospital type and comorbidities (table 1).

There were more female than male patients in this study. Prematching and postmatching results, including 30-day mortality and other complications, are reported in tables 2 and 3 . The overall 30-day mortality was $2.45 \%$, with no significant difference between the two groups before PSM. However, after propensity score analysis,

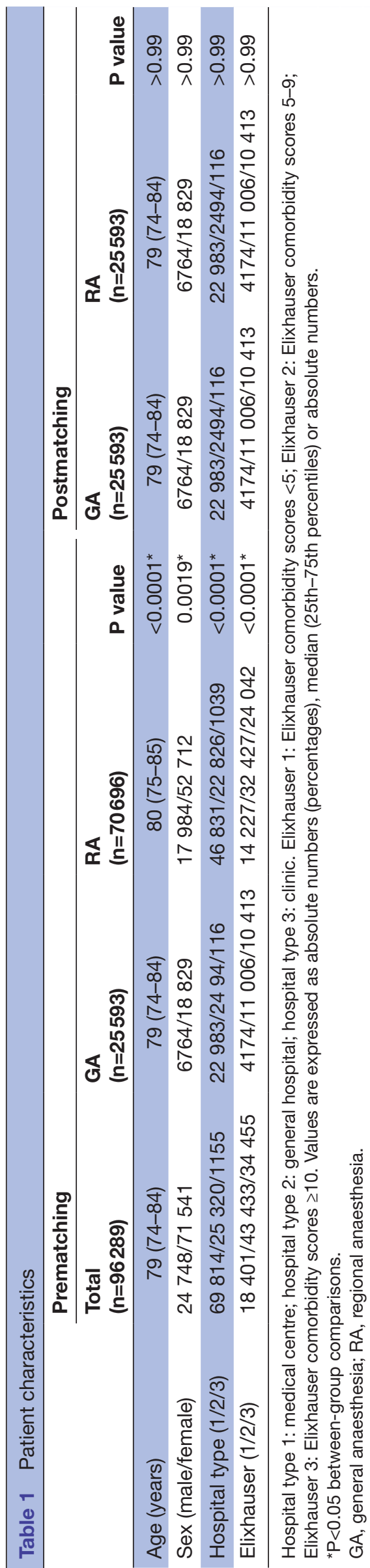


Table 2 Complications

\begin{tabular}{|c|c|c|c|c|c|c|c|}
\hline & \multicolumn{3}{|c|}{ Prematching ( $n=96289)$} & \multicolumn{4}{|c|}{ Postmatching $(n=51186)$} \\
\hline & $\begin{array}{l}G A \\
(n=25593)\end{array}$ & $\begin{array}{l}\text { RA } \\
(n=70696)\end{array}$ & $P$ value & $\begin{array}{l}G A \\
(n=25593)\end{array}$ & $\begin{array}{l}\text { RA } \\
(n=25593)\end{array}$ & $P$ value & $95 \% \mathrm{Cl}$ \\
\hline MI & $393(1.53)$ & $1504(2.13)$ & $<0.0001^{*}$ & $393(1.53)$ & $424(1.66)$ & 0.27 & 0.001 to 0.036 \\
\hline Pulmonary embolism & $1454(5.68)$ & $929(1.31)$ & $<0.0001^{*}$ & $1454(5.68)$ & $654(2.55)$ & $<0.0001^{*}$ & -0.011 to 0.014 \\
\hline Cerebral haemorrhage & $223(0.87)$ & $295(0.42)$ & $<0.0001^{*}$ & $223(0.87)$ & $123(0.48)$ & $<0.0001^{*}$ & -0.011 to 0.021 \\
\hline ARDS & $201(0.78)$ & $472(0.67)$ & 0.053 & $201(0.78)$ & $165(0.64)$ & 0.057 & -0.0063 to 0.036 \\
\hline Pulmonary oedema & $445(1.74)$ & $1244(1.76)$ & 0.83 & $445(1.74)$ & $451(1.76)$ & 0.84 & -0.0089 to 0.019 \\
\hline AKI & $655(2.56)$ & $1635(2.31)$ & $0.027^{\star}$ & $655(2.56)$ & $655(2.56)$ & $>0.99$ & -0.0019 to 0.028 \\
\hline Hepatic failure & $153(0.59)$ & $348(0.49)$ & 0.044 & $153(0.59)$ & $165(0.64)$ & 0.50 & -0.012 to 0.012 \\
\hline Sepsis & $273(1.07)$ & $637(0.90)$ & 0.019 & $273(1.07)$ & $263(1.02)$ & 0.66 & -0.010 to 0.019 \\
\hline
\end{tabular}

Values are expressed as absolute numbers (percentages) or absolute numbers.

${ }^{\star} \mathrm{P}<0.05$ between-group comparisons.

AKI, acute kidney injury; ARDS, acute respiratory distress syndrome; GA, general anaesthesia; MI, myocardial infarction; RA, regional anaesthesia.

the mortality was higher in the GA than in the RA group $(\mathrm{p}=0.0208)$.

Postoperative complications, including sepsis, hepatic failure, acute kidney injury and myocardial infarction, were not significantly different between the two groups after PSM, although the difference between the two groups was significant before PSM ( $\mathrm{p}=0.0189,0.0443$, 0.0265 and $<0.0001$, respectively). Other complications, including cerebral haemorrhage, pulmonary embolism, stay in the ICU, ventilator care, delirium and cost, differed significantly between the two groups before and after matching. These outcomes showed a higher incidence in the GA group than in the RA group. The overall cost of anaesthesia was also higher in the GA than in the RA group (prematching $\$ 5389.51$ vs $\$ 4919.75$, $\mathrm{p}<0.0001$; postmatching $\$ 5389.61$ vs $\$ 5213.24, \mathrm{p}<0.0001$ )

\section{DISCUSSION}

This study of nationwide claims data of 96289 patients confirmed that RA yielded a better outcome in terms of 30-day mortality than did GA for elderly patients undergoing surgery for hip fracture. Furthermore, delirium requiring pharmacological intervention was significantly lower in patients who received RA. Moreover, patients who received RA had a lower incidence of ICU admission, ventilator care, hospital costs and complications, including pulmonary embolism and cerebral haemorrhage.

Several observational studies ${ }^{7-12}$ have shown no benefit of RA compared with GA in patients with hip fractures. The meta-analyses performed by Mason et al and Guay showed that there was no significant difference between GA and RA regarding both postoperative delirium and long-term postoperative cognitive dysfunction. ${ }^{13}{ }^{14}$ The recent meta-analysis performed by Patel et $\mathrm{al}^{15}$ found no evidence of the influence of type of anaesthesia on postoperative delirium or mortality. However, our findings were similar to those of another national claims database study. ${ }^{3}$ Compared with these previous studies, the strength our study was that it included more recent data, from 2009 to 2015, with a large sample size. A previous study using Taiwan's inpatient claims database of geriatric patients between 1997 and 2011 reported that neuraxial anaesthesia offered some advantages in terms of mortality, stroke, respiratory failure, renal failure, hospital stay, ICU admission, mechanical ventilator support and medical costs. ${ }^{3}$ In that study, the primary outcome of mortality

Table 3 Primary and secondary outcomes

\begin{tabular}{|c|c|c|c|c|c|c|c|}
\hline & \multicolumn{3}{|c|}{ Prematching ( $n=96289)$} & \multicolumn{4}{|c|}{ Postmatching $(n=51186)$} \\
\hline & $\begin{array}{l}\text { GA } \\
(n=25593)\end{array}$ & $\begin{array}{l}\text { RA } \\
(n=70696)\end{array}$ & $P$ value & $\begin{array}{l}\text { GA } \\
(n=25593)\end{array}$ & $\begin{array}{l}R A \\
(n=25593)\end{array}$ & $P$ value & $95 \% \mathrm{Cl}$ \\
\hline Mortality & $654(2.55)$ & $1706(2.41)$ & 0.21 & $654(2.55)$ & $574(2.24)$ & $0.0047^{*}$ & -0.099 to 0.016 \\
\hline Delirium & $5828(22.77)$ & $12733(18.01)$ & $<0.0001^{*}$ & $5828(22.77)$ & $5187(20.27)$ & $<0.0001^{\star}$ & 0.019 to 0.045 \\
\hline ICU & 8055 (31.47) & $12683(17.94)$ & $<0.0001^{*}$ & $8055(31.47)$ & $5838(22.81)$ & $<0.0001^{*}$ & 0.046 to 0.070 \\
\hline Ventilator care & $1207(4.72)$ & $1095(1.55)$ & $<0.0001^{*}$ & 1207 (4.72) & 459 (1.73) & $<0.0001^{\star}$ & -0.0024 to 0.023 \\
\hline
\end{tabular}

Values are expressed as absolute numbers (percentages), median (25th-75th percentile) or absolute numbers.

${ }^{*} \mathrm{P}<0.05$ between-group comparisons.

GA, general anaesthesia; RA, regional anaesthesia; ICU, intensive care unit. 
was in-hospital mortality. However, we used a 30-day in or out of hospital mortality as a standard time frame that can directly reflect the level of hospital care. ${ }^{12}$ Our study also showed a lower incidence of ICU admission, reduced need for ventilator care, lower hospital costs and fewer complications, including pulmonary embolism and cerebral haemorrhage. Thus, in elderly patients, RA has some advantages.

Several randomised controlled trials (RCTs) have been conducted in an attempt to confirm the association between hip fracture and anaesthesia techniques. ${ }^{16}{ }^{17}$ According to O'Donnell's et al meta-analysis, 8200 patients per group would be required to achieve the minimum acceptable standard power $(80 \%)$ for detecting a difference with respect to 30 -day mortality. ${ }^{7}$ However, the number of patients in the two RCTs was only $45^{16}$ and 322 , respectively. ${ }^{17}$ Although RCTs are not clinically or financially easy to perform, several pragmatic large RCTs assessing the effectiveness of GA and RA are underway. The RAGA-delirium trial is planned with 1000 randomised older patients undergoing hip fracture surgery. ${ }^{18}$ The REGAIN and iHope studies are international, multicentre, pragmatic RCTs including 1600 and 1032 patients, respectively. ${ }^{19}{ }^{20}$ Moreover, the REGARD study comparing RA and GA and their effect on delirium in patients with hip fractures aiming to recruit 100 patients has been registered in the ISRCTN registry (ISRCTN1565914, https://doi.org/10.1186/ ISRCTN15165914). A meta-analysis of their results may support our study findings and thus improve guideline development for enhanced outcome after hip fracture surgery. ${ }^{19} 20$

Delirium is used as an indicator of healthcare quality for elderly patients. Identification of vulnerable patients and prevention of and intervention for delirium in hip fracture are of great public health concern. ${ }^{21}$ Delirium occurrence in the ICU is associated with a 2-4 fold increase in the mortality rates. ${ }^{22-25}$ Postoperative delirium is very common and has an impact on the length of stay in the postanaesthesia care unit and mortality and has been identified as an independent risk factor for in-hospital mortality and for mortality during 6 months of follow-up. ${ }^{25}$ Several studies have identified GA as a risk factor for delirium. In contrast, Smith et $a l^{26}$ have reported that intraoperative variables, including type of anaesthesia, were not associated with delirium. However, their study was a meta-analysis based on only 6704 patients. Therefore, our results may more reliably reflect the occurrence of delirium in elderly patients with hip fracture.

Delirium, an acute disorder of attention and cognition, is a clinical condition that is often unrecognised and overlooked and is difficult to evaluate. ${ }^{4}$ It comprises 23 ICD-10 codes, which represent the difficulty of the diagnosis, and the sensitivity for delirium is only approximately $3 \%{ }^{27}$ In clinical settings, where screening instruments are not used systematically, diagnosis is missed in approximately $60 \%-80 \%$ of cases. ${ }^{28}$ It has seldom been evaluated with nationwide claims data because of the lack of accuracy in defining delirium in a wide-incidence range. ${ }^{7}$ Therefore, we needed to use a working definition to ensure the accuracy of diagnosis of postoperative delirium.

There are various methods to treat delirium, including ensuring patient safety with psychiatric, environmental and supportive management and identifying and treating the cause of delirium. Regarding pharmacological interventions, antipsychotic medication has been considered as the first-line pharmacotherapy of delirium except in cases of sedative or alcohol withdrawal. ${ }^{29}$ Among the antipsychotic drugs, haloperidol is most frequently employed, while risperidone and quetiapine are increasingly being used. Haloperidol, risperidone and quetiapine have shown comparable efficacy and safety in the treatment of delirium. ${ }^{30}$ Therefore, in our study, we defined 'delirium' based on a record of prescription of intravenous haloperidol, risperidone or quetiapine. This definition of delirium was established based on the delirium-treatment guideline of the American Psychiatric Association. ${ }^{31}$ However, according to our definition, the diagnosis of the hypoactive subtype of delirium may have been missed as the use of drugs in hypoactive delirium remains controversial. ${ }^{32}$ Although it remains unclear whether the hyperactive or mixed subtype of delirium could pose a more serious problem than the hypoactive subtype, patients with hyperactive delirium are more likely to experience falls, ${ }^{33}$ intravenous line disconnection or incidental trauma in clinical settings. Hence, we used a definition of delirium that presents with symptoms requiring clinical interventions.

The strength of our study is that it was based on a large and representative national database that is widely used as a reliable research tool for identifying trends and demographics. A large-scale database can offer a vast amount of information, which can help improve the quality of medical care. Administrative claims databases are more useful for the evaluation of long-term complications, and patients can often be tracked within the time period of the databases for assessment of specific events. They are more representative of the general population. ${ }^{34} \mathrm{We}$ also used the Elixhauser Comorbidity Index, which is a more recent model that includes 31 conditions. ${ }^{35}$ There are various risk stratification tools for predicting patient mortality and morbidity, such as the Charlson model and Nottingham Hip Fracture Score; therefore, future studies should focus on other risk scoring models. ${ }^{36}$ We also used the ICD-10 codes that were introduced to enhance and expand the ICD-9-CM. ${ }^{37}$ Furthermore, we used more recent data (2009-2015) to reflect improvements in anaesthetic drugs, perioperative haemodynamic monitoring and perioperative pain management.

\section{Study limitations}

This study had some limitations. First, this was a retrospective national claims data investigation, and thus, the patients' clinical data, such as body weight, perioperative blood loss and duration of surgery, were not included due to the nature of the database. To minimise heterogeneity, 
we only included femoral neck fracture (S720) or trochanteric fracture (S721). Second, claims data can contain coding errors. Third, information on diagnosis and disease included in the healthcare utilisation database may not have sufficient validity for identifying disease occurrence and prevalence, since the data have not been established for research purposes but rather for medical services claims and reimbursement. Fourth, as previously stated, the definition of delirium in our study has limitations. The initial treatment of delirium involves multidisciplinary non-pharmacological strategies such as reorientation, family presence and interaction, minimising disturbances in the sleep-wake cycle and addressing sensory impairment with hearing aids and eyeglasses, noise reduction and mobilisation. ${ }^{28}$ Delirium not requiring pharmacological intervention may have missed detection in this study. Because of these limitations, we can only determine the association between the type of anaesthesia and mortality and not causal relationships. Therefore, the data and results require careful interpretation.

\section{CONCLUSIONS}

We conducted a retrospective analysis of the data of 96289 elderly patients with hip fracture. RA was associated with better outcomes in terms of mortality and delirium than GA in elderly patients who underwent hip fracture surgery. Additionally, compared with GA, RA was associated with better outcomes in terms of ICU admission, ventilator care, hospital costs and complications, including pulmonary embolism and cerebral haemorrhage in elderly patients with hip fracture surgery.

\section{Author affiliations}

${ }^{1}$ Department of Anesthesiology and Pain Medicine, Inje University Seoul Paik Hospital, Seoul, Republic of Korea

${ }^{2}$ Department of Anesthesiology and Pain Medicine, Chung-Ang University College of Medicine and Graduate School of Medicine, Seoul, Republic of Korea

Acknowledgements This study used NHIS-NSC data (NHIS-2017-1-234) made by National Health Insurance Service (NHIS).

Contributors EJA contributed to data collection, data analysis, data interpretation and writing of the manuscript. HJK contributed to the literature review, study design and reviewed the manuscript. KWK contributed to figure and table preparation and reviewed the manuscript. HRC contributed to data analysis and writing of the manuscript. HK contributed to data interpretation and reviewed the manuscript. SRB contributed to the literature review, figure preparation, study design, data collection, analysis and interpretation and writing of the manuscript.

Funding This research was supported by the Basic Science Research Program through the National Research Foundation of Korea (NRF), funded by the Ministry of Education (NRF-2017R1D1A3B03034298).

Competing interests The authors declare no conflict of interest with the NHIS.

Patient consent for publication Not required.

Ethics approval The study was reviewed and approved by the institutional review board of Seoul Paik Hospital (IRB No 2018-04-007).

Provenance and peer review Not commissioned; externally peer reviewed.

Data availability statement Data may be obtained from a third party and are not publicly available.
Open access This is an open access article distributed in accordance with the Creative Commons Attribution Non Commercial (CC BY-NC 4.0) license, which permits others to distribute, remix, adapt, build upon this work non-commercially, and license their derivative works on different terms, provided the original work is properly cited, appropriate credit is given, any changes made indicated, and the use is non-commercial. See: http://creativecommons.org/licenses/by-nc/4.0/.

\section{REFERENCES}

1. YC H, Park YG, Nam KW, et al. Trend in hip fracture incidence and mortality in Korea: a prospective cohort study from 2002 to 2011 . Korean Med Sci 2015;30:483-8.

2. YC H, Kim TY, Lee A, et al. Current trends and future projections of hip fracture in South Korea using nationwide claims data. Osteoporos Int 2016;27:2603-9.

3. Chu C-C, Weng S-F, Chen K-T, et al. Propensity Score-matched comparison of postoperative adverse outcomes between geriatric patients given a general or a neuraxial anesthetic for hip surgery. Anesthesiology 2015;123:136-47.

4. Inouye SK, Westendorp RGJ, Saczynski JS. Delirium in elderly people. The Lancet 2014;383:911-22.

5. Seitz DP, Gill SS, Bell CM, et al. Postoperative medical complications associated with anesthesia in older adults with dementia. $J$ Am Geriatr Soc 2014;62:2102-9.

6. Menendez ME, Neuhaus V, van Dijk CN, et al. The Elixhauser comorbidity method outperforms the Charlson index in predicting inpatient death after orthopaedic surgery. Clin Orthop Relat Res 2014;472:2878-86.

7. Basques BA, Bohl DD, Golinvaux NS, et al. General versus spinal anaesthesia for patients aged 70 years and older with a fracture of the hip. Bone Joint J 2015;97-B:689-95.

8. Neuman MD, Rosenbaum PR, Ludwig JM, et al. Anesthesia technique, mortality, and length of stay after hip fracture surgery. JAMA 2014;311:2508-17.

9. O'Hara DA, Duff A, Berlin JA, et al. The effect of anesthetic technique on postoperative outcomes in hip fracture repair. Anesthesiology 2000;92:947-57.

10. White SM, Moppett IK, Griffiths R. Outcome by mode of anaesthesia for hip fracture surgery. An observational audit of 65535 patients in a national dataset. Anaesthesia 2014;69:224-30.

11. Brox WT, Chan PH, Cafri G, et al. Similar mortality with general or regional anesthesia in elderly hip fracture patients. Acta Orthop 2016;87:152-7.

12. Tung $\mathrm{Y}-\mathrm{C}, \mathrm{Hsu} \mathrm{Y}-\mathrm{H}$, Chang G-M. The effect of anesthetic type on outcomes of hip fracture surgery: a nationwide population-based study. Medicine 2016;95:e3296.

13. Mason SE, Noel-Storr A, Ritchie CW. The impact of general and regional anesthesia on the incidence of post-operative cognitive dysfunction and post-operative delirium: a systematic review with meta-analysis. J Alzheimers Dis 2010;22:S67-79.

14. Guay J. General anaesthesia does not contribute to long-term postoperative cognitive dysfunction in adults: a meta-analysis. Indian $J$ Anaesth 2011;55:358-63.

15. Patel V, Champaneria R, Dretzke J, et al. Effect of regional versus general anaesthesia on postoperative delirium in elderly patients undergoing surgery for hip fracture: a systematic review. BMJ Open 2018;8:e020757.

16. Biboulet $P$, Jourdan $A$, Van Haevre $V$, et al. Hemodynamic profile of target-controlled spinal anesthesia compared with 2 target-controlled general anesthesia techniques in elderly patients with cardiac comorbidities. Reg Anesth Pain Med 2012;37:433-40.

17. Parker MJ, Griffiths R. General versus regional anaesthesia for hip fractures. A pilot randomised controlled trial of 322 patients. Injury 2015;46:1562-6.

18. Li T, Yeung J, Li J, et al. Comparison of regional with general anaesthesia on postoperative delirium (RAGA-delirium) in the older patients undergoing hip fracture surgery: study protocol for a multicentre randomised controlled trial. BMJ Open 2017;7:e016937.

19. Neuman MD, Ellenberg SS, Sieber FE, et al. Regional versus general anesthesia for promoting independence after hip fracture (REGAIN): protocol for a pragmatic, international multicentre trial. BMJ Open 2016;6:e013473.

20. Kowark A, Adam C, Ahrens J, et al. Improve hip fracture outcome in the elderly patient (iHOPE): a study protocol for a pragmatic, multicentre randomised controlled trial to test the efficacy of spinal versus general anaesthesia. BMJ Open 2018;8:e023609.

21. Yang $\mathrm{Y}, \mathrm{Z}$ hao $\mathrm{X}$, Dong T, et al. Risk factors for postoperative delirium following hip fracture repair in elderly patients: a systematic review and meta-analysis. Aging Clin Exp Res 2017;29:115-26. 
22. Ely EWet al. Delirium as a predictor of mortality in mechanically ventilated patients in the intensive care unit. JAMA 2004;291:1753-62.

23. Lin S-M, Liu C-Y, Wang C-H, et al. The impact of delirium on the survival of mechanically ventilated patients*. Crit Care Med 2004;32:2254-9.

24. van den Boogaard M, Schoonhoven L, van der Hoeven JG, et al. Incidence and short-term consequences of delirium in critically ill patients: a prospective observational cohort study. Int J Nurs Stud 2012;49:775-83.

25. Veiga $\mathrm{D}$, Luis $\mathrm{C}$, Parente $\mathrm{D}$, et al. Postoperative delirium in intensive care patients: risk factors and outcome. Rev Bras Anestesiol 2012;62:469-83.

26. Smith TO, Cooper A, Peryer G, et al. Factors predicting incidence of post-operative delirium in older people following hip fracture surgery: a systematic review and meta-analysis. Int $J$ Geriatr Psychiatry 2017;32:386-96.

27. Inouye SK, Leo-Summers L, Zhang Y, et al. A chart-based method for identification of delirium: validation compared with interviewer ratings using the confusion assessment method. J Am Geriatr Soc 2005;53:312-8.

28. Safavynia SA, Arora S, Pryor KO, et al. An update on postoperative delirium: clinical features, neuropathogenesis, and perioperative management. Curr Anesthesiol Rep 2018;8:252-62.

29. Lacasse H, Perreault MM, Williamson DR. Systematic review of antipsychotics for the treatment of hospital-associated delirium in medically or surgically III patients. Ann Pharmacother 2006;40:1966-73.

30. Yoon H-J, Park K-M, Choi W-J, et al. Efficacy and safety of haloperidol versus atypical antipsychotic medications in the treatment of delirium. BMC Psychiatry 2013;13:240.

31. American psychiatric association. Practice guideline for the treatment of patients with delirium. Am J Psychiatry 1999;156:1-20.

32. Fong TG, Tulebaev SR, Inouye SK. Delirium in elderly adults: diagnosis, prevention and treatment. Nat Rev Neurol 2009;5:210-20.

33. O'Keeffe S. Clinical significance of delirium subtypes in older people. Age Ageing 1999;28:115-9.

34. Bedard NA, Pugely AJ, McHugh MA, et al. Big data and total hip arthroplasty: how do large databases compare? J Arthroplasty 2018;33:41-5.

35. Elixhauser A, Steiner C, Harris DR, et al. Comorbidity measures for use with administrative data. Med Care 1998;36:8-27.

36. Marufu TC, Mannings A, Moppett IK. Risk scoring models for predicting peri-operative morbidity and mortality in people with fragility hip fractures: qualitative systematic review. Injury 2015;46:2325-34.

37. Quan $\mathrm{H}$, Sundararajan V, Halfon $\mathrm{P}$, et al. Coding algorithms for defining comorbidities in ICD-9-CM and ICD-10 administrative data. Med Care 2005;43:1130-9. 